\title{
Turning nutrition on its head - Noakes gets his day
}

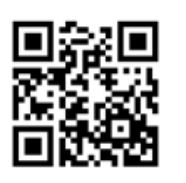

Indefatigable, with a conviction to match his harshest critics, Prof. Tim Noakes took to the scientific trenches in Cape Town in February, fusing the firepower of 15 of the world's top experts on the low-carbohydrate, high-fat (LCHF) diet at a heavily subscribed R3 million, 4-day 'health convention'.

Nutritionists, cardiologists, surgeons, family physicians and researchers, many of them best-selling authors, rallied to his side, presenting findings, analyses, arguments on causation, and alarming, undisputed obesity and type 2 diabetes prevalence studies to counter last year's unprecedented local wave of scientific antipathy towards Noakes much of it from his own colleagues at the University of Cape Town (UCT)'s Faculty of Health Sciences.

Nearly all the international speakers came at short notice and at their own expense, rounded up by Karen Thomson, granddaughter of heart surgeon Prof. Chris Barnard, who began a unique sugar/ carbohydrate addiction clinic after winning a battle with alcohol and cocaine addiction. Thomson wanted to give the charismatic and popular sports scientist a platform to defend himself after what he described to Izindaba as a 'kangaroo court' UCT Centenary debate 15 months ago in which he took on fellow UCT graduate and fellow A-rated scientist Prof. Jacques Rossouw, Washington-based epidemiologist, researcher on heart disease prevention, and former director of the South African Medical Research Council's Institute for Nutritional Diseases. Noakes encountered what he described as a hostile audience there, and saw the debate as 'a set-up', declining to engage further after he and his adversary had outlined their initial arguments. A top academic in the chronic disease field, plus a leading professor of endocrinology and diabetic medicine at UCT (Profs Krisela

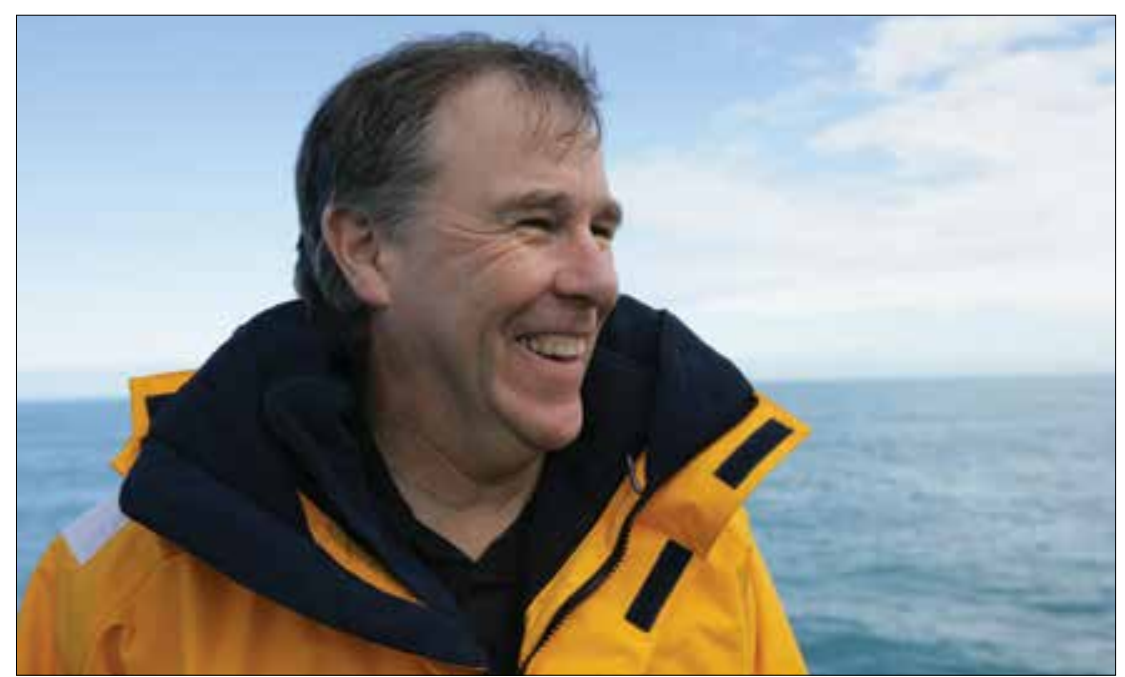

Prof. Tim Noakes.

Steyn and Dinky Levitt, respectively), later labelled Noakes' revolutionary approach to nutrition as 'a public health threat and extremely dangerous', accusing him of having 'a superficial understanding of epidemiology'. UCT's Faculty of Health Sciences then took the unprecedented step of publicly slamming Noakes' dietary advice, claiming that he was making 'outrageous unproven' claims about disease prevention and 'maligning the integrity and credibility of peers who criticise his diet for being evidence-deficient and not conforming to the tenets of good and responsible science?. 'This goes against UCT's commitment to academic freedom as the prerequisite to fostering responsible and respectful intellectual debate and free enquiry', the statement added. Noakes offered an olive branch in his closing remarks at the February health convention, describing his local colleagues as 'honest, genuine people who spent their lives trying to help people and speaking out of deep sense of conviction. However, he remains unyielding: 'We take the position that they are wrong.' He said that the health convention was the direct result of SAMPs coverage of the UCT centenary debate and his critics' comments there.

\section{Consensual position outlined}

The unanimous and urgent viewpoint distillation in last month's long-awaited counteroffensive was as simple as it was dramatic: mainstream nutritional and dietary advice has not only failed dismally, it is the immediate cause of the global obesity and diabetes epidemics. It completely ignores the history of why and how human nutrition developed over the last 3 million years, and more importantly it refuses to acknowledge the presence of insulin resistance (carbohydrate resistance) as the single most prevalent biological state in modern humans eating according to current dietary guidelines promoting low fat and high carbohydrate intakes. People with insulin resistance were at an increased risk of developing a wide range of chronic medical conditions if they ingested high-carbohydrate diets for any length of time (i.e. decades).' Every speaker at the convention signed this consensual position statement. 


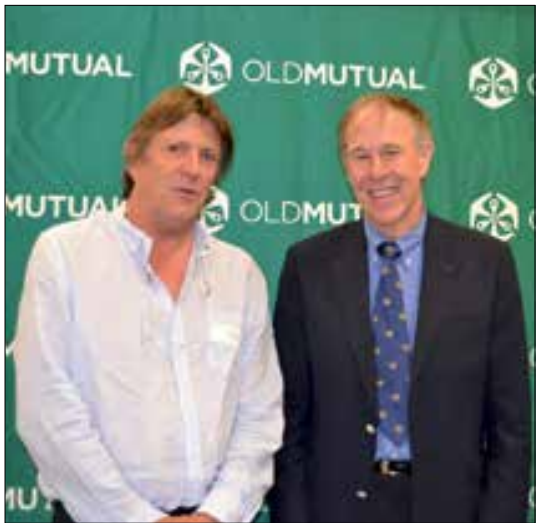

Dr Peter Bond and Prof. Noakes.

Noakes said that conventional scientific enquiry had 'dropped the bar so low that we came to the point where anything can cause anything', describing the proponents of conventionally accepted nutrition as 'wanting more funding to do more research they're not necessarily interested in getting you healthier. Parading several formerly obese Capetonians, virtually unrecognisable after their dramatic weight loss on the LCHF diet, Noakes said all their maladies, including atrial fibrillation, type 2 diabetes, sleep apnoea, hypertension, gout and dry flaky skin, had disappeared. One convert, Brian Berkman, a type 2 diabetic, said he was on the verge of undergoing bariatric surgery when he decided to try the Banting diet as a last resort - with spectacular and unexpected success.

\section{Speakers 'risked their careers' by taking a stand}

Noakes commented: 'We are surrounded by a population that is full of insulin resistance and we're not doing anything about it.' $\mathrm{He}$ said that none among his array of highcalibre speakers had any conflict of interests, describing them as 'having put their careers on the line because they were too honest'. They were prepared to 'stand up against the world and say this cannot go on forever'. American science writer and physicist Gary Taubes, the best-selling author of Why We Get Fat and Good Calories, Bad Calories, told his Cape Town audience that the Cochrane Collaboration research in 2002 showed that weight loss achieved in calorie-restricted diets was 'so small as to be clinically insignificant'. He added: 'If we are obese, eat less and end up fat anyway, it's a pretty good sign that eating less doesn't work. Clinical trials prove it. Exercising more also doesn't work.' Citing the American Heart Association and American College of Sports Medicine physical activity guidelines of 2007, he continued: 'It's reasonable to assume that persons with relatively high daily energy expenditures would be less likely to gain weight, compared with those who have low energy expenditures. So far, data to support this hypothesis are not particularly compelling. He said these 100 - 150-year-old hypotheses had been shown not to work in centuries of testing, thinking and randomised controlled trials. 'The idea of energy-in minus energy-out, perverted appetite and eating too much with insufficient expenditure is supposedly based on the first law of thermodynamics. It doesn't tell you anything about why people get fat. It's nonsensical'.

\section{Obesity 'a hormonal regulatory disorder'}

Taubes' alternative hypothesis, founded on his research, was that obesity was a hormonal regulatory disorder. Like type 2 diabetes, obesity was fundamentally a disorder of insulin signalling, hence the term 'diabesity'. The carbohydrate content of the diet triggered it with the key driver of insulin being the carboyhdrates and sugar in the diet, not fat and not saturated fat. Obesity was shown to be a disorder of excess fat accumulation (not energy balance, not over-eating and not sedentary behaviour). People did not need an excess of food to become obese. Mice made fat out of their food under 'the most unlikely circumstances, even when half-starved. Every animal experiment he'd ever found showed that animals did not get fat by over-eating. The degree of insulin resistance or sensitivity was a key factor in the equation of fat accumulation, and explained why diabetes type 2 and obesity went together. More and more research was proving that 'carbohydrate is driving insulin is driving fat'. Taubes said that the solution to obesity and its associated epidemics was not getting people to move more and eat less, but restricting the causative agent - i.e. refined grains and sugars.

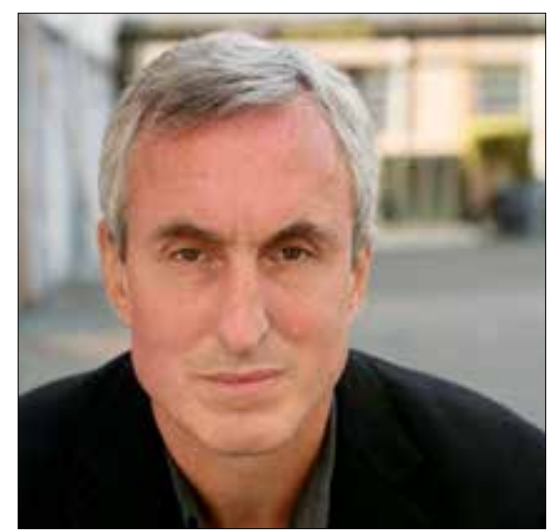

Gary Taubes, best-selling author

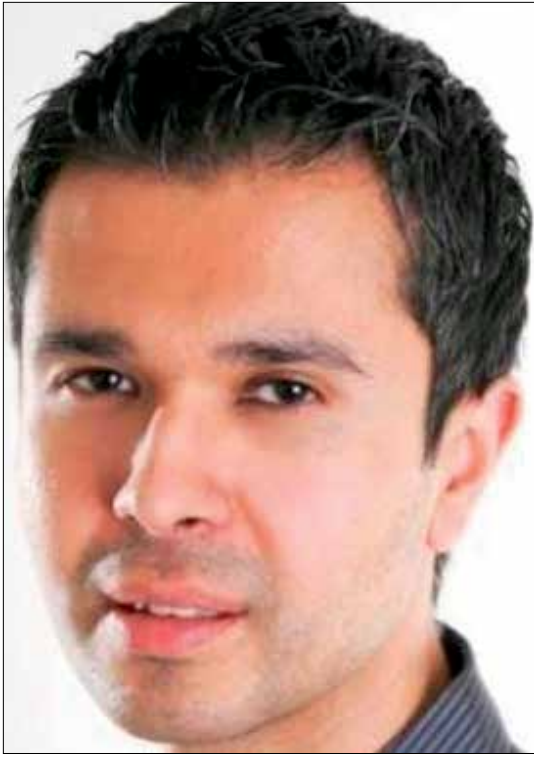

UK cardiologist Dr Aseem Malhotra.

Tax sugary drinks, subsidise low-carb/ high-fat foods

Cardiologist Dr Aseem Malhotra, from the UK, accused the food industry of 'spiking' food with hidden sugar, making it impossible to avoid it. He advocated a tax on sugary drinks and subsidisation of healthy alternatives low in carbs and high in fat. Dr Stephen Phinney, a North American physician/scientist who has spent 35 years studying diet, exercise, fatty acids and inflammation, said it would be in the interests of governments to find a dietary solution for the poor who existed mostly on the 'two absolute no-no's of sugar and flour'. Sweden's most popular health blogger, family medicine specialist, Dr Andreas Eenfeldt, said it was 'no coincidence' that the prevalence of obesity and type 2 diabetes had 'shot up' over the past 30 years since the dietary guidelines of the 1980s advocated high-carb, low-fat diets. Swedes' consumption of butter had doubled over the past two decades, yet heart attacks had dropped over the same period, an anomaly not explained by conventional diet proponents. The Swedish government, after complaints about advocates of LCHF nutrition, had conducted a high-level probe resulting in Sweden becoming the first nation to reject the low-fat diet dogma and adopt the LCHF diet for treatment of people with obesity. Studies across the globe were showing that low-carb diets were not only compatible with the scientific evidence but best practice for overweight or obese patients - with no evidence of harm having emerged. He said that the most popular weight-loss Google searches in Sweden from 2008 to date were LCHF, low glycaemic index, the Paleo diet and Weight Watchers. By the end of 2010 
the LCHF diet was already in 'a league of its own', and by 2015 it was more popular than all the other weight-loss diets combined. A recent survey had shown that $23 \%$ of the Swedish population was now on some kind of low-carb diet.

The health convention was sponsored by Old Mutual, whose chief medical officer Dr Peter Bond told delegates that the world's most rapidly spreading and sustained pandemic was not HIV/AIDS but diseases of lifestyle and diet, of which obesity was the most dominant. He said that at current rates the world's obese population would outnumber those suffering from starvation by 2025. Insurance loadings in South Africa (SA) were increasing all the time, with a $17 \%$ deterioration in sub-standard lives over the past 4 years. He estimated that 3.5 million South Africans had type 2 diabetes, and that 5 million were insulin resistant or had impaired fasting glucose or impaired glucose tolerance. Only half of Old Mutual's clients were aware that they had these conditions, and people were often only diagnosed during an insurance examination.

\section{Undiagnosed diabetes heralds CVD epidemic}

Bond cited a 2012 study in a coloured community in Bellville, Cape Town, ${ }^{[1]}$ showing the prevalence of type 2 diabetes to be $28.2 \%$, with $88 \%$ of affected females and $42 \%$ of affected males being obese according to waist measurement. A full 55\% were undiagnosed, despite nearby healthcare facilities. Bond said this indicated that the prevalence of diabetes had increased hugely in the coloured community. The high prevalence of undiagnosed diabetes 'portends that cardiovascular diseases might grow to epidemic proportions in the near future in SA' SA already had the highest overweight and obesity rate in sub-Saharan Africa, with 7 out of 10 women and 4 out of 10 men overweight or obese, double the global rate of nearly $30 \%$. Not a single country had managed to reduce its obesity rate in the past three decades, he emphasised.

Noakes described the epidemic of obesity and diabetes as 'the greatest modern threat to human health', adding that 'we need to front up and admit that we have been wrong for the past 40 years and must now change'. $\mathrm{He}$ hoped the convention which Chris Barnard had inadvertently inspired would help grant the famous heart surgeon one of his most fervent wishes: that had he focused on preventive medicine earlier he would have saved 150 million lives, rather than the 150 patients via heart transplantation.

\section{Chris Bateman}

chrisb@hmpg.co.za

S Afr Med J 2015;105(3):248-250.

DOI:10.7196/SAMJ.9554
1. Erasmus RT, Soita DJ, Hassan MS, et al. High prevalence of diabetes mellitus and metabolic syndrome in a South African coloured popu- lation: Baseline data of a study in Bellville, Cape Town. S Afr Med

